\title{
Méthodes photothermiques : contrôle non destructif et spectroscopie
}

\author{
A.C. Boccara et D. Fournier* \\ Laboratoire d'Optique Physique de l'ESPCI, UPR A0005 du CNRS, ESPCI, 10 rue \\ Vauquelin, 75005 Paris, France \\ * Laboratoire d'Instrumentation de l'UPMC, UPR A0005 du CNRS, ESPCI, 10 rue \\ Vauquelin, 75005 Paris, France
}

\begin{abstract}
Résumé : l'objet de ce chapitre est d'introduire dans l'instrumentation optique les méthodes de mesure en régime non stationnaire (modulé ou impulsionnel). Pour illustrer ces méthodes, nous avons choisi différentes applications des détections photothermiques dans les domaines de la caractérisation thermique, de la mesure des défauts subsurfaciques et de la spectroscopie de systèmes échappant aux techniques conventionnelles de spectrophotometrie.
\end{abstract}

Dans le chapitre consacré à la lumière polarisée, nous avons décrit un certain nombre de méthodes (ellipsométrie, interférométrie) susceptibles de révéler des structures ou des hétérogénéités de très faible dimension (par rapport à la longueur d'onde de la lumière) qui se situaient dans la gamme du nanomètre au picomètre. Rappelons que la sensibilité de ces méthodes d'interférences en lumière polarisée tenait au fait que les deux composantes du champ qui interféraient, suivaient des chemins identiques (ou parfois très voisins) à travers les structures sondées évitant ainsi les effets des dérives thermiques ou mécaniques des montages.

Dans ce chapitre nous examinerons encore les performances de montages optiques susceptibles de mesurer de très faibles différences de marche, mais celles-ci seront induites par des stimulations non stationnaires (modulées ou impulsionnelles). Ces phénomènes se produisent sur des temps suffisamment brefs pour que les dérives n'affectent pas les signaux mesurés ; ceux-ci étant de plus, périodiques, ou répétitifs, on pourra avantageusement utiliser les moyens traditionnels de traitement du signal (détection synchrone, moyenneur...).

Pour illustrer ces méthodes de détection, nous nous baserons principalement sur la description des effets photothermiques (élévation de température associée à l'absorption d'un flux lumineux instationnaire) et de quelques unes de leurs applications dans le domaine du contrôle non destructif et de la spectroscopie d'absorption d'échantillons "exotiques", c'est-à dire dont les coefficients d'absorption sont trop importants (objets opaques) ou trop faibles (objets très transparents) pour être étudiés par les méthodes spectrophotométriques traditionnelles. Par la suite nous nous limiterons aux seules détections optiques des effets photothermiques, mais d'autres effets peuvent être utilisés pour révéler ce que nous définirons par la suite comme des "ondes thermiques" (figure 1).

\section{SIGNAUX ET BRUITS : QUELQUES ORDRES DE GRANDEUR}

Dans tous les montages que nous décrirons, c'est la variation du flux du faisceau sonde qui portera l'information sur la mesure. Aussi est-il bon de se poser les questions suivantes :

- Quels sont les paramètres physiques qui affectent la sensibilité de la mesure? 
- Quelles précautions faut-il prendre pour que les limites physiques de la mesure soient atteintes?

- Quelles configurations expérimentales paraissent les mieux adaptées à la mesure pour obtenir le meilleur rapport performances/complexité ou performances/prix ?

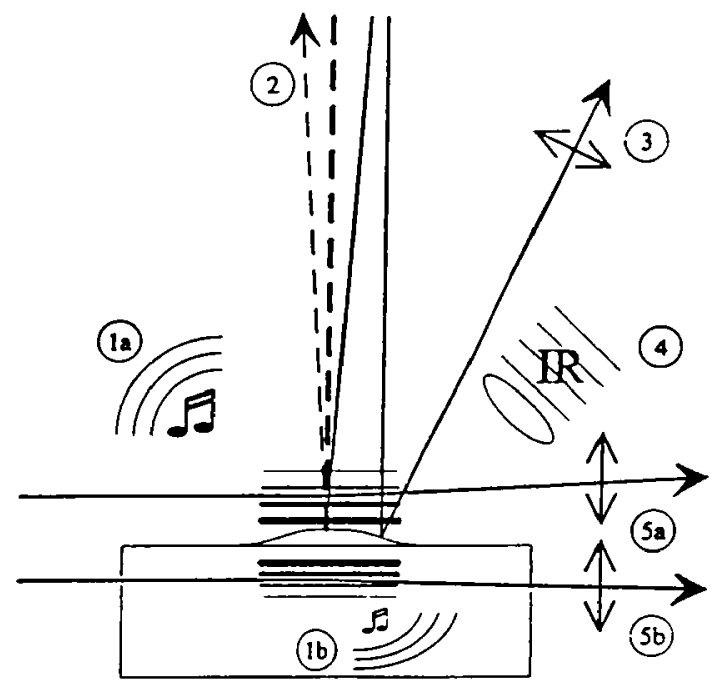

Figure 1 : Un flux lumineux absorbé à la surface d'un échantillon crée une onde thermique dans l'échantillon et provoque un grand nombre d'effets physiques qui peuvent être exploités pour déterminer les propriétés thermiques et optiques : une onde sonore est créée dans le gaz entourant l'échantillon (1a) ou dans son volume (1b) (effet photo-acoustique) ; la réflectivité de surface est modulée, ce qui peut être détecté en mesurant lá partie modulée d'un faisceau laser sonde réfléchi à la surface $(2)$; l'onde thermique cause une dilatation thermique de l'échantillon qui périodiquement dévie ou déphase un faisceau sonde réfléchi à la surface (3); le rayonnement infrarouge émis par l'échantillon peut être capté par un détecteur (4); la modification de l'indicc de réfraction du gaz à la surface de l'échantillon (5) ou dans son volume peut être mesuré par la déflexion d'un faisceau laser sonde (effet mirage).

Rappelons que, lorsqu'un faisceau de puissance moyenne $\Phi$ tombe sur un détecteur quantique pour créer un courant $\overline{\mathfrak{i}}$, les fluctuations associées au "bruit de photons" (appelé aussi bruit de granulation, bruit Shot ou bruit Schottky) sont données par l'écart quadratique moyen :

$\overline{\mathrm{di}}_{\mathrm{p}}^{2}=2 \mathrm{e} \overline{\mathrm{i}} \Delta \mathrm{f}$ (e charge de l'électron, $\Delta \mathrm{f}$ bande passante du système de détection)

D'autres bruits peuvent affecter la mesure, comme les bruits thermiques de résistance :

$$
\overline{\mathrm{di}}_{\mathrm{t}}^{2}=\frac{4 \mathrm{kT}}{\mathrm{R}} \Delta \mathrm{f}
$$

Mais dans les conditions des expériences que nous décrirons (fort flux dont on cherchera à mesurer une faible variation), on peut généralement se placer dans le cas où le bruit de 
photons est dominant. Essayons de prévoir quelles limites correspondent à ce bruit pour quelques expériences que nous utiliserons plus loin au cours de ce chapitre.

\subsection{Photoréflectance}

On irradie un échantillon de réflectivité $R$ avec un faisceau sonde de flux $\Phi$; dans ce cas on enregistre les variations périodiques $\Phi d R$ du flux $\Phi R$ du faisceau sonde réfléchi par la surface de l'échantillon (lui même chauffé localement). $R$ étant le facteur de réflexion, $d R$ sa variation induite dont la variation minimum détectable a pour valeur :

$$
\left(\frac{\mathrm{dR}}{\mathrm{R}}\right)_{\min }=\frac{\sqrt{\mathrm{di} \overline{\mathrm{i}}^{2}}}{\overline{\mathrm{i}}}=\frac{\sqrt{2 \mathrm{e} \Delta \mathrm{f}}}{\sqrt{\overline{\mathrm{i}}}}
$$

pour $0,5 \mathrm{~mW}$ sur la diode, et une sensibilité de $0,25 \mathrm{~A} / \mathrm{W}$ (par exemple du silicium détectant une radiation de longueur d'onde égale à $0,6 \mu \mathrm{m})$ on trouve $\left(\frac{\mathrm{dR}}{\mathrm{R}}\right)_{\min }=0,510^{-7} / \sqrt{\mathrm{Hz}}$.

En pratique on s'aperçoit que cette performance assez spectaculaire peut être obtenue avec des sources incohérentes et des détecteurs de faible coût (LED $\approx 10 \mathrm{~F}$ et photodiode $\approx 10$ à $20 \mathrm{~F}$ à l'unité) et que les sources lasers (HeNe ou diodes laser à semiconducteurs) sont beaucoup trop bruyantes, au moins dans certaines gammes de fréquences, pour nous permettre d'atteindre ces sensibilités.

\subsection{Interférométrie}

Dans ce cas, ce sont les variations de la différence de marche qui induisent une variation de flux à la sortie de l'interféromètre (à deux ondes : par exemple un interféromètre de Michelson dont un miroir oscille).

Si le flux émergent $\Phi$ est donné par :

$$
\phi=\frac{\phi_{\mathrm{o}}}{2}\left(1+\cos 2 \pi \frac{\delta}{\lambda}\right)
$$

sa variation aura pour valeur :

$$
\mathrm{d} \phi=-\phi_{\mathrm{o}} \frac{\pi}{\lambda} \sin \left(\frac{2 \pi \delta}{\lambda}\right) \mathrm{d} \delta
$$

En reprenant les chiffres ci-dessus pour la variation $\mathrm{d} \Phi / \Phi\left(0,510^{-7} \mathrm{~Hz}^{-1 / 2}\right)$ et en prenant $\Phi=\frac{\Phi_{0}}{2}$ (flux moyen qui correspond au maximum de sensibilité lorsque $\frac{2 \pi \delta}{\lambda}=\frac{\pi}{2} \pm \mathrm{k} \pi$ :

$$
\mathrm{d} \Phi / \Phi=2 \frac{\pi}{\lambda} \mathrm{d} \delta \text { et }(\mathrm{d} \delta)_{\min }=\frac{\lambda}{2 \pi} 0,510^{-7} \approx 0,610^{-14} \mathrm{~m} / \sqrt{\mathrm{Hz}}
$$

Pour atteindre ces performances, indépendamment des considérations de stabilité du flux déjà discutées, il faut prendre de sérieuses précautions pour isoler le montage des perturbations qui peuvent affecter la différence de marche (vibrations, convections...). 


\subsection{Photodéflexion}

Il s'agit ici de mesurer la déviation d'un faisceau sonde (par exemple dans un gradient d'indice associé à un gradient de température).

La méthode la plus simple consiste à envoyer le faisceau (flux $\phi$ ) sur deux diodes adjacentes. Si le diamètre (supposé uniforme) du faisceau est $2 \mathrm{a}$, une variation $\mathrm{dx}$ de la position du faisceau induira une augmentation du flux sur l'une des diodes de $\Phi_{0} \frac{4}{\pi} \frac{\mathrm{dx}}{\mathrm{a}}$ et une diminution identique sur l'autre diode.

Comme les angles sont faibles, la mesure de la différence des signaux $\left(\Phi_{\circ} \frac{8}{\pi} \frac{d x}{a}\right)$ est proportionnelle à la déviation cherchée. diode

Pour un faisceau de divergence $\theta$ avec un détecteur au silicium et un flux de $0,5 \mathrm{~mW}$ par

$$
\begin{gathered}
(\delta \theta)_{\min } \approx 10^{-8} \theta \quad \text { soit pour une valeur typique } \theta=10^{-2} \mathrm{rd} \\
(\delta \theta)_{\min } \approx 10^{-10} \mathrm{rad} / \sqrt{\mathrm{Hz}} .
\end{gathered}
$$

Remarquons que le fait de travailler avec la différence de deux signaux issus d'une même source conduit à une très forte réjection du bruit d'intensité ce qui est très utile lorsque des lasers sont utilisés comme sonde.

\section{LES ONDES THERMIQUES}

Ce terme décrit la solution de l'équation de diffusion de la chaleur dans un espace (ou un demi-espace) homogène :

$$
\rho c \frac{\partial T}{\partial t}=k \Delta T
$$

où $\rho$ est la masse volumique, $\mathrm{c}$ la chaleur spécifique et $\mathrm{k}$ la conductivité thermique.

Lorsque la source est un plan $(x, y)$ en $z=0$ et lorsque l'on impose des conditions de flux périodique (pulsation $\omega=2 \pi \mathrm{f}$ ), la partie modulée de la température dans le demi-espace $z \geq 0$ prendra la forme :

$$
T=T_{0} \exp (-z / \mu) \cos (\omega t-z / \mu)
$$

$\mu$ est la longueur de diffusion thermique (qui est sensiblement la longueur sur laquelle la chaleur diffuse pendant une période de modulation) et s'écrit :

$$
\mu=\sqrt{2 \mathrm{k} / \rho \mathrm{c \omega} \omega}=\sqrt{2 \mathrm{Dt} / \omega}
$$

où $\mathrm{D}_{\mathrm{t}}$ est la diffusivité thermique du milieu.

Le tableau ci-après donne quelques exemples de valeurs de $\mu$ pour différents matériaux à différentes fréquences de modulation. 
$\mu$ pour différentes fréquences de modulation et différents matériaux

\begin{tabular}{|l|l|llll|}
\multicolumn{1}{c|}{} & \multicolumn{4}{c|}{} & \\
\cline { 2 - 5 } \multicolumn{1}{c|}{} & $\mathrm{D}_{\mathbf{i}}\left(\mathrm{cm}^{2} / \mathrm{s}\right)$ & $10 \mathrm{~Hz}$ & $1 \mathrm{kHz}$ & $100 \mathrm{kHz}$ & $10 \mathrm{MHz}$ \\
$\mathrm{Air}$ & 0.9 & $1.7 \mathrm{~mm}$ & $0.17 \mathrm{~mm}$ & $17 \mu \mathrm{m}$ & $1.7 \mu \mathrm{m}$ \\
$\mathrm{CCl}_{4}$ & 0.2 & $0.8 \mathrm{~mm}$ & $0.08 \mathrm{~mm}$ & $8.0 \mu \mathrm{m}$ & $0.8 \mu \mathrm{m}$ \\
& $7 \times 10^{-4}$ & $0.047 \mathrm{~mm}$ & $4.7 \mu \mathrm{m}$ & $0.47 \mu \mathrm{m}$ & $0.047 \mu \mathrm{m}$ \\
\hline
\end{tabular}

Lorsque la source de chaleur est ponctuelle (en $x=y=z=0$ ) elle engendre une onde thermique sphérique ayant la forme:

$$
T(r)=\frac{A}{r} \exp (-r / \mu) \cos (\omega t-r / \mu)
$$

où $\mathrm{A}$ est une constante et $\mathrm{r}^{2}=\mathrm{x}^{2}+\mathrm{y}^{2}+\mathrm{z}^{2}$.

\subsection{Détection des ondes thermiques}

Nous ne retiendrons que les méthodes optiques qui ont l'avantage de fonctionner sans contact matériel avec l'échantillon, possèdent une bonne sensibilité $\left(10^{-2}\right.$ à $\left.10^{-7} \mathrm{~K} / \sqrt{\mathrm{Hz}}\right)$ et couvrent des échelles spatiales allant du $\mathrm{mm}$ au $\mu \mathrm{m}$.

La détection "mirage" (figure 2):

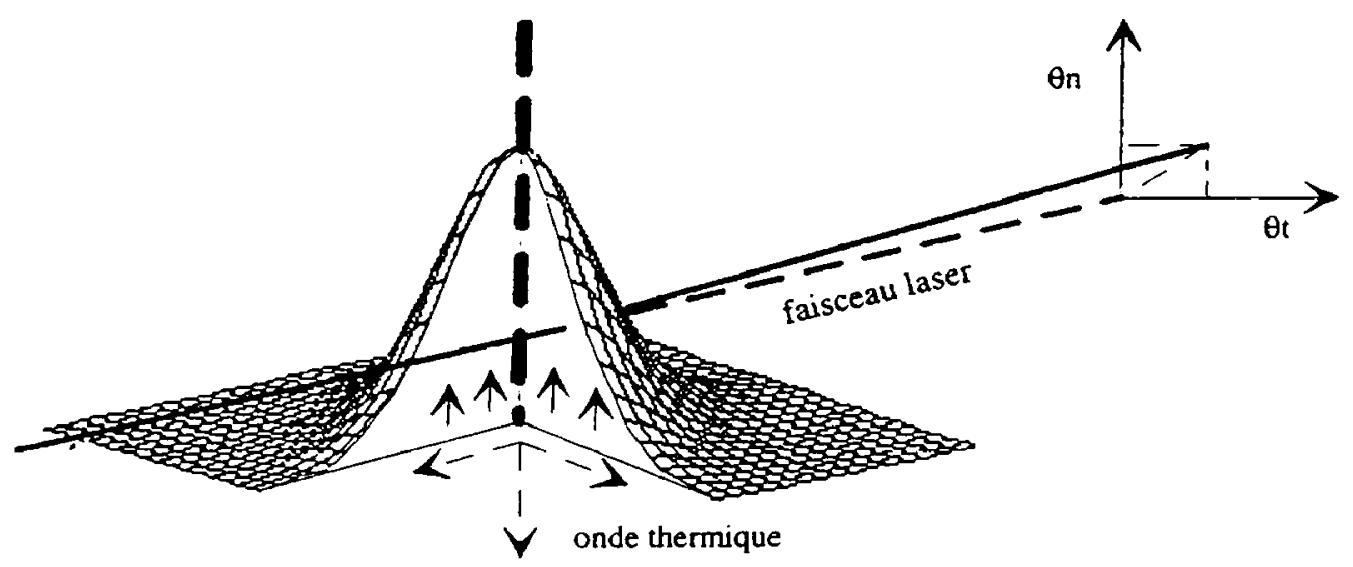

Figure 2 : Principe de l'effet mirage : un faisceau laser est défléchi par un champ thermique modifiant l'indice de réfraction

Il s'agit d'utiliser le gradient périodique de température donc d'indice (n) qui règne à la surface d'un échantillon chauffé périodiquement (à cause de la forme amortie de l'onde 
thermique) pour dévier un faisceau sonde se propageant par exemple le long de la surface de l'échantillon dans l'air ou dans un fluide dans lequel baigne l'échantillon.

La déviation $\theta$ a pour expression, pour un gradient uniforme dans la direction $z$ et une longueur d'interaction $L$ :

$$
\theta=\frac{L}{n} \frac{d n}{d z}=\frac{L}{n} \frac{d n}{d T} \frac{d T}{d z}
$$

Comme $\frac{\mathrm{dn}}{\mathrm{dT}} \approx 10^{-6}$ pour l'air et $5 \cdot 10^{-4}$ pour un liquide très transparent comme le tétrachlorure de carbone $\mathrm{CCl}_{4}$, avec un $\theta_{\min } \approx 10^{-10} \mathrm{rad} / \sqrt{\mathrm{Hz}}$ on peut mesurer des élévations périodiques de température de $10^{-5} \mathrm{~K} / \sqrt{\mathrm{Hz}}$ dans l'air à quelques $10^{-8} \mathrm{~K} / \sqrt{\mathrm{Hz}}$ dans $\mathrm{CCl}_{4}$ pour des valeurs de $L$ de l'ordre du $\mathrm{cm}$.

La photoréflectance (figure 3) :

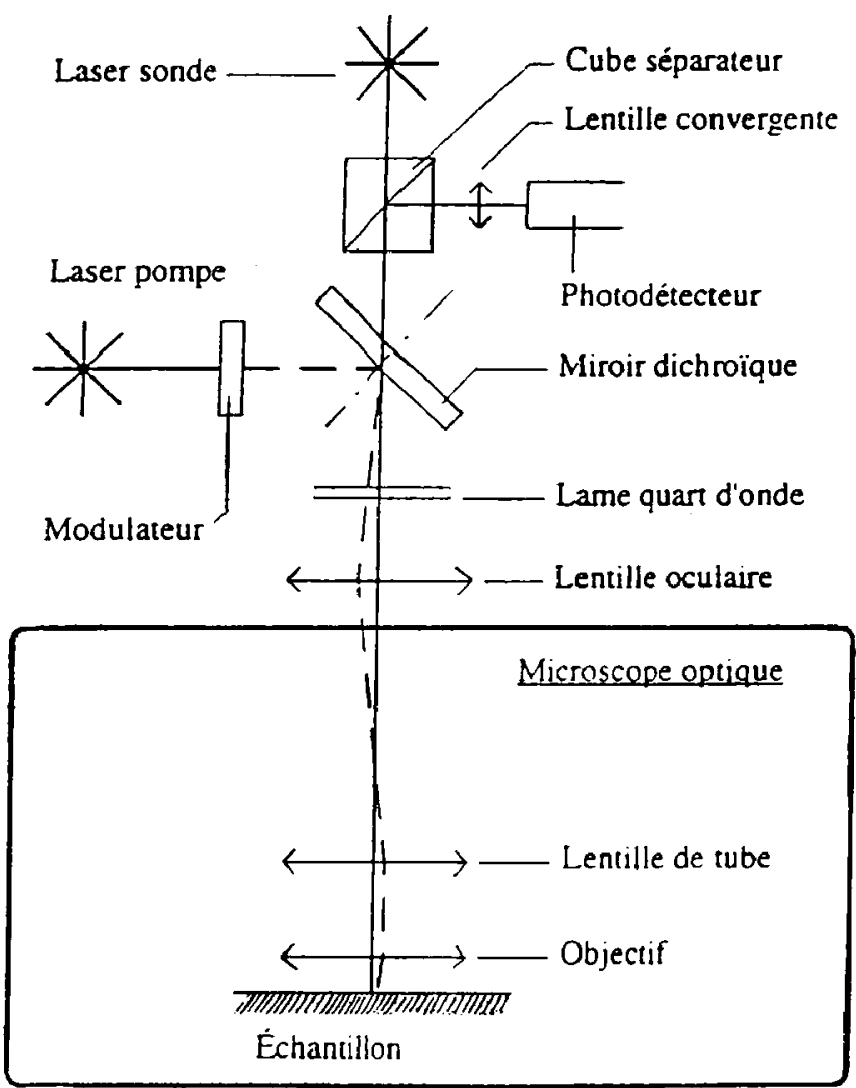

Figure 3 : Schéma du microscope photothermique fonctionnant par photoréflectance. 
Bien que très sensible, l'effet mirage ne permet pas d'opérer avec une résolution spatiale inférieure à quelques dizaines de micromètres. Si on cherche à explorer le champ de température à la surface d'un échantillon d'une façon plus locale, il est possible de focaliser un faisceau sonde à travers un microscope jusqu'à des dimensions de l'ordre du micromètre et d'utiliser la variation de réflectivité :

$$
\frac{\Delta \mathrm{R}}{\mathrm{R}}=\frac{1}{\mathrm{R}} \frac{\mathrm{dR}}{\mathrm{dT}} \Delta \mathrm{T}
$$

$\frac{\mathrm{dR}}{\mathrm{dT}}$ est de l'ordre de $10^{-5}$ à $10^{-6 / K}$ pour les matériaux les plus courants (métaux, semiconducteurs...), comme $\left(\frac{\Delta R}{R}\right)_{\min }$ est de l'ordre de $10^{-6}$ à $10^{-7}$ on peut espérer $(\Delta T)_{\min }$ $=10^{-1}$ à $10^{-3} \mathrm{~K} / \sqrt{\mathrm{Hz}}$.

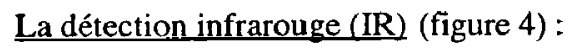

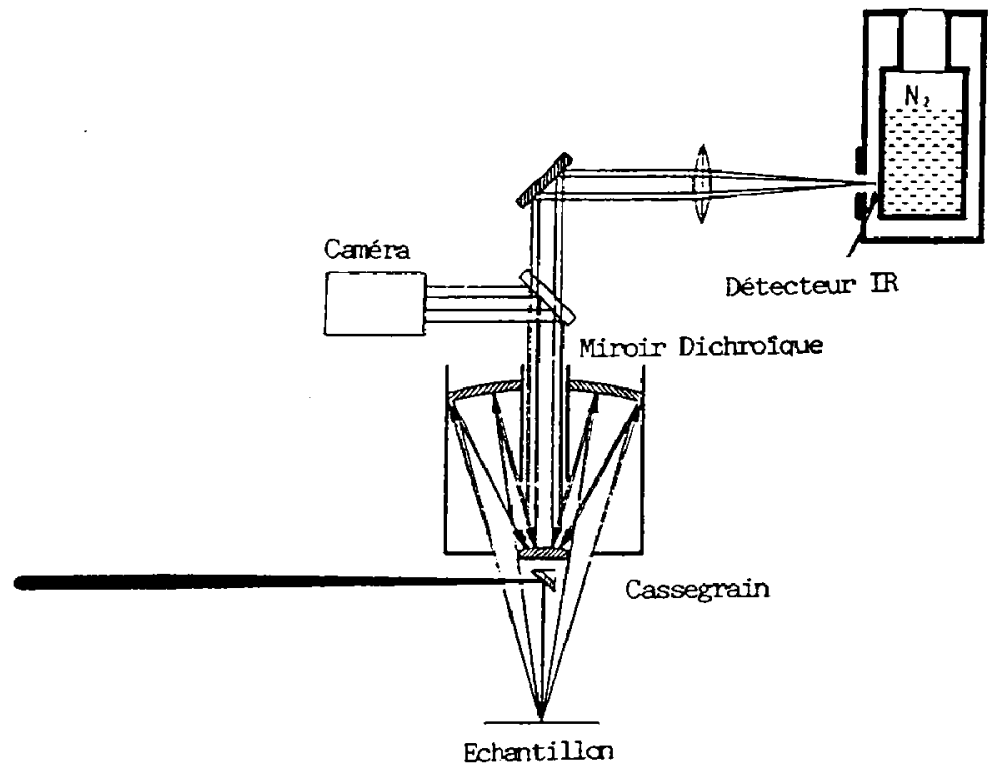

Figure 4 : Détection infrarouge de l'élévation de température locale

Dans ce cas on mesure le flux infrarouge modulé émis par l'échantillon. À température ambiante, c'est dans l'infrarouge autour de $10 \mu \mathrm{m}$ que se situe le maximum d'émission, aussi des détecteurs de type HgCdTe sont-ils bien adaptés à ce type de mesure. Comme l'émittance est

$$
P=\varepsilon \sigma T^{4}
$$

sa variation s'écrit :

$$
\mathrm{dP}=4 \varepsilon \sigma \mathrm{T}^{3} \mathrm{dT}
$$


où $\varepsilon$ est l'émissivité et $\sigma=5,6710^{-12} \mathrm{~W} / \mathrm{cm}^{2} . \mathrm{K}^{-4}$ est la constante de Stéfan.

Ce mode de détection permet d'atteindre pour de bons émetteurs infrarouge $(\varepsilon \approx 1)$ des variations de température de l'ordre de $10^{-4} \mathrm{~K} / \sqrt{\mathrm{Hz}}$ mais il est difficile d'utiliser une détection infrarouge pour étudier des métaux comme l'aluminium ou le cuivre qui sont très bons réflecteurs (donc de mauvais émetteurs $(\varepsilon \approx 0)$ ) dans cette région du spectre.

\subsection{Les ondes thermiques planes aux interfaces}

Nous avons vu l'expression de l'onde thermique dont la structure ressemblait à celle d'une onde électromagnétique plane qui se propagerait dans un milieu absorbant. En fait, compte tenu de la forme particulière de l'équation de diffusion le vecteur d'onde complexe vaut

$$
(1+\mathrm{i}) / \mu
$$

Que se passe-t-il lorsqu'une telle "onde" rencontre une interface entre deux milieux 0 et 2 dont les propriétés thermiques sont différentes ? La réponse est donnée (comme en électromagnétisme) en écrivant des équations de continuité à l'interface, continuités de la température et du flux

$$
T_{0}=T_{2} \text { et }-k_{0} \frac{d T_{0}}{d z}=-k_{2} \frac{d T_{2}}{d z}
$$

( $\mathrm{z}$ direction de la normale). On trouve un facteur de réflexion

$$
r_{o 2}=\left(\left(k_{o} \rho_{0} C_{o}\right)^{1 / 2}-\left(k_{2} \rho_{2} C_{2}\right)^{1 / 2}\right) /\left(\left(k_{o} \rho_{o} C_{o}\right)^{1 / 2}+\left(k_{2} \rho_{2} C_{2}\right)^{1 / 2}\right)
$$

et de transmission

$$
t_{\mathrm{o} 2}=2\left(\mathrm{k}_{\mathrm{o}} \rho_{\mathrm{o}} \mathrm{C}_{\mathrm{o}}\right)^{1 / 2} /\left(\left(\mathrm{k}_{\mathrm{o}} \rho_{\mathrm{o}} \mathrm{C}_{\mathrm{o}}\right)^{1 / 2}+\left(\mathrm{k}_{2} \rho_{2} \mathrm{C}_{2}\right)^{1 / 2}\right)
$$

Remarquons que ces facteurs sont réels (ce qui se produirait aussi pour des ondes électromagnétiques se propageant dans des milieux où les parties réelle $n$ et imaginaire $k$ de l'indice sont égales). La quantité

$$
e_{i}=\left(k_{i} p_{i} C_{i}\right)^{1 / 2}
$$

joue le rôle de l'indice en optique et s'appelle l'effusivité thermique. Notons, qu'à la différence des indices de réfraction, les effusivités peuvent varier de plusieurs ordres de grandeur et les facteurs de réflexion sont alors voisins de 1.

Nous avons vu, dans le domaine de l'optique, comment les interférences qui se produisent dans une couche mince déposée à la surface du matériau affectent les amplitudes réfléchies ou transmises. Que se passe-t-il pour les ondes thermiques si une couche d'épaisseur $I_{1}$ (faible devant $\mu_{1}$ ) vient perturber l'interface entre les deux milieux (infinis) 0 et 2 ?

Le facteur de réflexion prend alors la forme :

$$
r=\frac{e_{0}-e_{2}+\sqrt{i \omega}\left(l_{1} / k_{1}\right)\left(e_{0} e_{2}-e_{1}^{2}\right)}{e_{0}+e_{2}+\sqrt{i \omega}\left(l_{1} / k_{1}\right)\left(e_{0} e_{2}+e_{1}^{2}\right)}
$$


La quantité $\frac{l_{1}}{k_{1}}$ représente la résistance thermique de la couche d'interface. Là encore la perturbation (à épaisseur $l_{1}$ constante) sera d'autant plus marquée que les propriétés thermiques $\left(e_{i}\right)$ des milieux 0 et 2 sont différentes de celle du milieu 1 (par exemple, comme on le verra plus tard une lame d'air même très fine apporte une très forte perturbation entre deux milieux métalliques).

\section{LE CONTRÔLE NON DESTRUCTIF DES MATÉRIAUX ET DES COMPOSANTS}

\subsection{Caractérisations thermiques}

La forme particulièrement simple des ondes thermiques dans un milieu homogène fournit un moyen très simple, par exploration du champ de température, de mesure de la longueur de diffusion thermique donc de la diffusivité thermique.

D'un point de vue pratique le déphasage $(\tau / \mu)$ qui existe entre la source et la zone sondée à une distance $r$ de la source est une grandeur directement accessible à partir d'une détection synchrone. La phase, à la différence de l'amplitude, est insensible aux fluctuations d'intensité de la source et aux éventuelles variations des propriétés optiques de l'échantillon (absorption, réflexion...).

$\grave{A}$ titre d'exemple, la figure 5 représente un champ de température de symétrie quasi "sphérique" qui est engendré par un spot de taille $1 \mu \mathrm{m}$ chauffant un échantillon de diamant monocristallin à la fréquence de $1 \mathrm{MHz}$. Les lignes isophases seraient des cercles correspondant à la phase $\frac{r}{\mu}$ (pour $r>1 \mu \mathrm{m}$ ) si le matériau était parfaitement homogène. Cette mesure a été faite par photoréflectance à l'aide du microscope photothermique décrit plus haut.

À une échelle différente l'effet mirage a été utilisé avec succès pour de nombreux matériaux dont le diamant polycristallin, des céramiques fonctionnelles, des matériaux composites etc., de structure isotrope ou anisotrope.

\subsection{Mesures d'épaisseurs}

Les expressions que nous avons données pour les facteurs de réflexion aux interfaces entre deux milieux et pour une lame mince en surface d'un matériau, nous montrent l'extrême dépendance de la température de surface avec les paramètres thermiques ou géométriques des différents milieux. Les effets géométriques sont d'autant plus marqués que les propriétés thermiques sont différentes.

Une application pratique de ces effets concerne la mesure des épaisseurs de couches minces métalliques, on peut par exemple mesurer avec une précision d'une fraction de nanomètre l'épaisseur d'un dépôt de titane ou de WSi à la surface du silicium.

Pour illustrer un peu plus quantitativement ces effets, la figure 6 représente les profils de phase du signal thermique calculés à différentes fréquences pour une couche d'or de $38 \mathrm{~nm}$ déposée sur de la silice. On pourrait penser qu'au moins à très basse fréquence, où la longueur de diffusion thermique de l'or est près de 500 fois plus grande que son épaisseur, la couche $n$ 'affecterait pas la diffusion thermique en surface. Il n'en n'est rien, comme le montre le tableau des valeurs calculées pour la longueur de diffusion thermique du système verre couche et on peut rendre compte de cette augmentation du "poids" de la couche d"or en la considérant comme un interféromètre à ondes multiples de type Fabry Perot, fonctionnant bien sûr à l'ordre 0 (épaisseur $<2 \pi \mu$, longueur d'onde thermique) et de finesse :

$$
\mathrm{F}=\pi \sqrt{\mathrm{r}_{01} \mathrm{r}_{12}} /\left(1-\mathrm{r}_{01} \mathrm{r}_{12}\right) \approx 50
$$


( $\sqrt{\mathrm{k} \rho \mathrm{c}}=5,8$ pour l'air, 28000 pour l'or, 900 pour la silice)

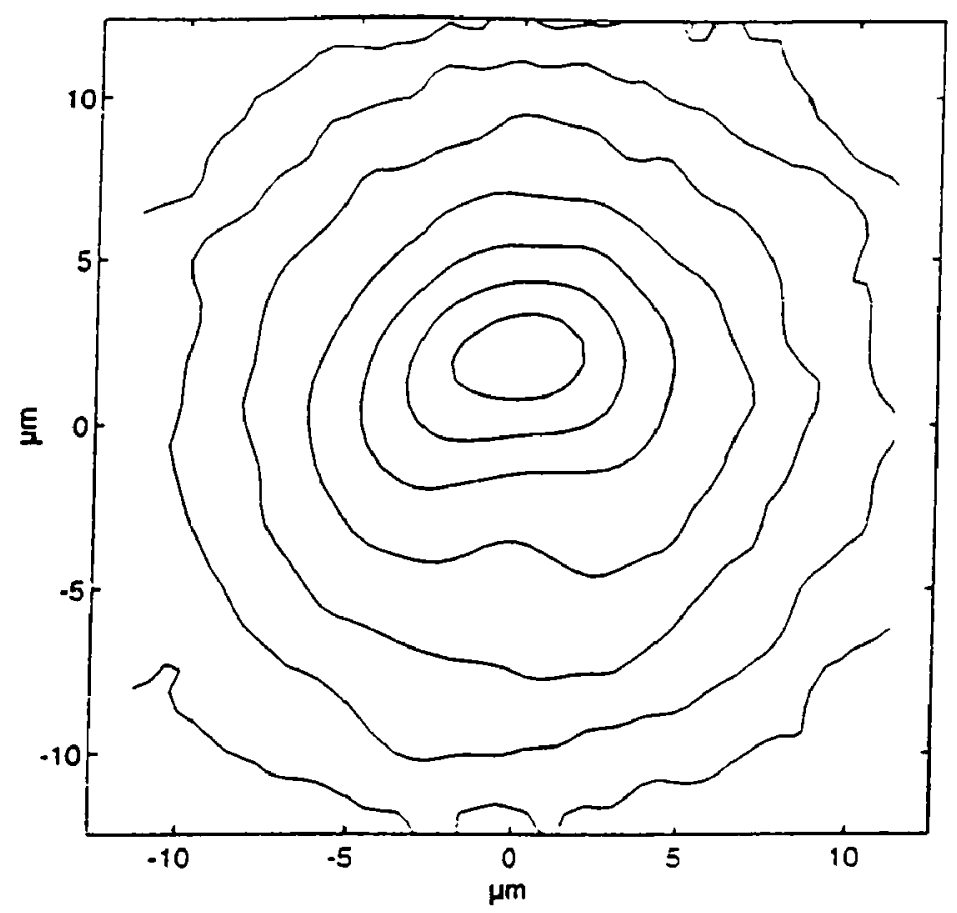

Figure 5 : Carte des isophases pour une mesure sur un échantillon de diamant monocristallin. L'écart entre les lignes est de $10^{\circ}$.

Cette finesse importante a pour effet d'augmenter la quantité de chaleur stockée dans la couche et donc de ralentir le transfert thermique.

Ces effets d'interférences multiples thermiques doivent nous faire penser à ce que nous avons déjà rencontré en ellipsométrie où des couches très minces devant la longueur d'onde optique perturbent considérablement les signaux à l'interface entre deux milieux.

\subsection{Défauts subsurfaciques, délaminations, fissures}

La partie modulée de la température de surface d'un échantillon peut également révéler l'existence de discontinuités thermiques pourvu que ces dernières ne se trouvent pas à une profondeur plus grande que la longueur de diffusion thermique $\mu$. En effet au delà de cette distance, l'onde thermique réfléchie serait complètement atténuée et son influence sur la température de surface serait négligeable. 

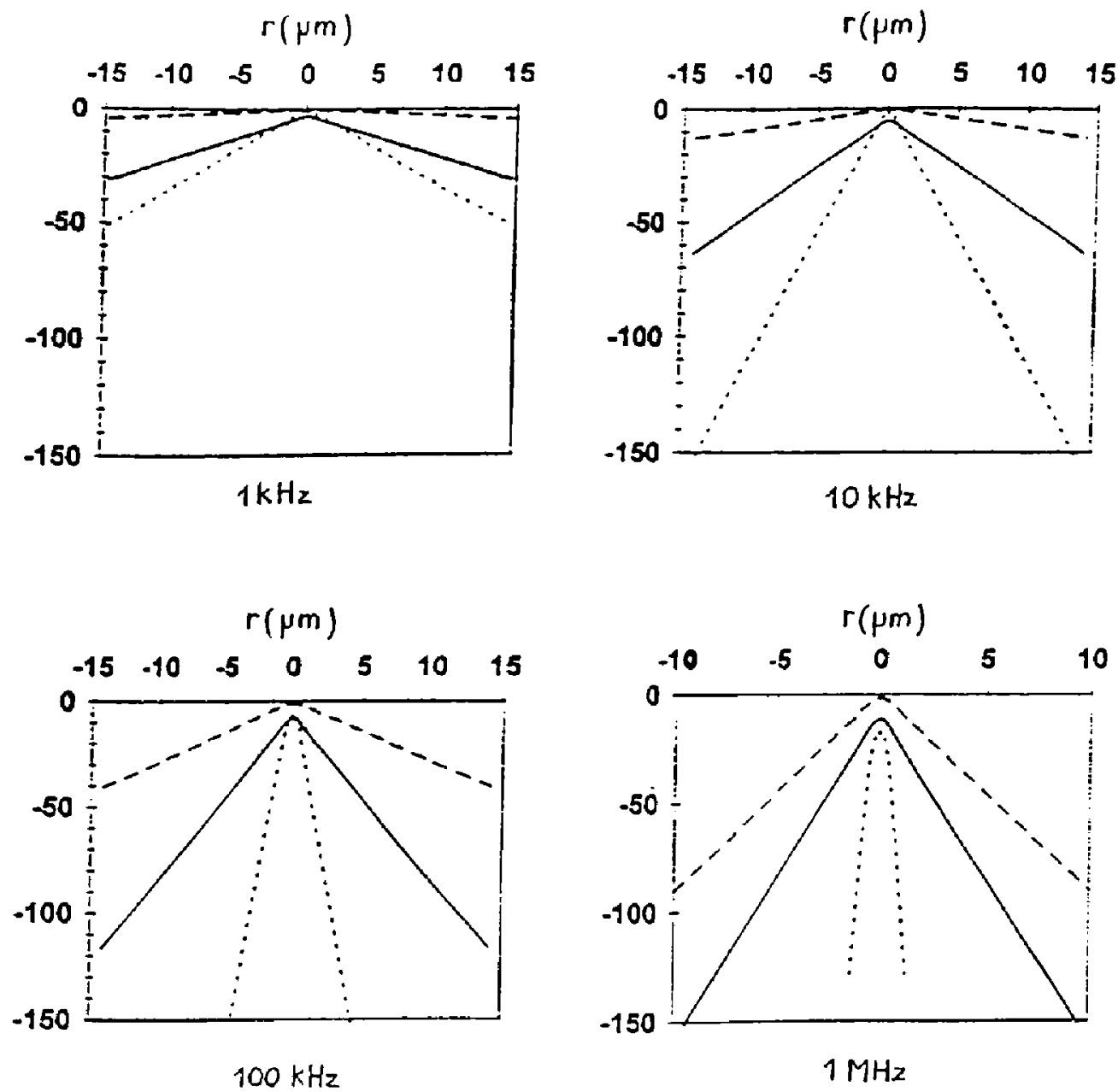

\begin{tabular}{|c|c|c|c|c|}
\hline & $1 \mathrm{kHz}$ & $10 \mathrm{kHz}$ & $100 \mathrm{kHz}$ & $1 \mathrm{MHz}$ \\
\hline$\mu_{\mathrm{or}}$ & $200 \mu \mathrm{m}$ & $63 \mu \mathrm{m}$ & $20 \mu \mathrm{m}$ & $6.3 \mu \mathrm{m}$ \\
\hline$\mu_{\mathrm{SiO2}}$ & $16 \mu \mathrm{m}$ & $5 \mu \mathrm{m}$ & $1.6 \mu \mathrm{m}$ & $0.5 \mu \mathrm{m}$ \\
\hline$\mu_{\mathrm{eff}}$ & $26 \mu \mathrm{m}$ & $12 \mu \mathrm{m}$ & $6.4 \mu \mathrm{m}$ & $3.4 \mu \mathrm{m}$ \\
\hline
\end{tabular}

Figure 6 : Profils de la phase de la température pour (-_..) $38 \mathrm{~nm}$ d'or sur silice, (-... -.) de l'or massif et (- - -) de la silice massive. 


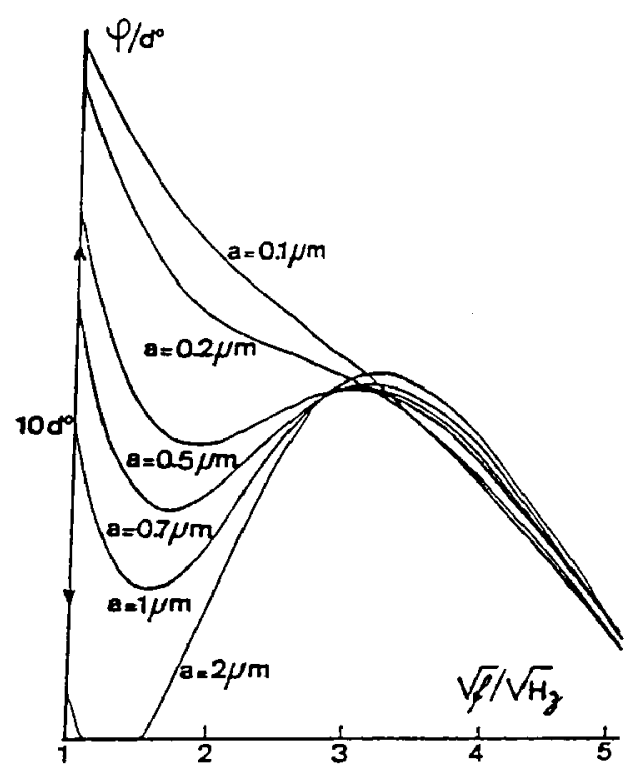

Figure 7 : Signal d'effet mirage en fonction de l'épaisseur du défaut (voir texte).

La figure 7 montre la variation en fréquence $(\sqrt{\mathrm{f}})$ du signal "mirage" pour un faisceau sonde situé à $0,15 \mathrm{~mm}$ de la surface d'une pièce en titane qui aurait une délamination d'épaisseur variant entre 0,1 et $2 \mu \mathrm{m}$ et située à $0,9 \mathrm{~mm}$ sous la surface. Cette figure appelle quelques commentaires :

- Le défaut est invisible au dessus de $9 \mathrm{~Hz}$ car à cette fréquence l'onde thermique issue de la surface, réfléchie sur le défaut et venant interférer avec l'onde incidente, a une amplitude négligeable.

- Dans la zone où le signal est bien révélé (environ $2 \mathrm{~Hz}$ ), l'écart est linéaire en fonction de l'épaisseur du défaut pour les faibles épaisseurs, puis au delà de $1 \mu \mathrm{m}$ le signal "sature" ce qui signifie qu'au delà de quelques $\mu \mathrm{m}$ le défaut se comporte comme si son épaisseur était infinie.

C'est en fabriquant un montage basé sur ce principe que les défauts de contact de bitubes de titane servant d'échangeurs thermiques de sous-marins nucléaires ont été mesurés. Notons que l'épaisseur de la lame d'air équivalente au défaut devait être inférieure à $0,35 \mu \mathrm{m}$ pour ne pas affecter la qualité de l'échangeur.

$\hat{A}$ une autre échelle cette méthode est utilisée pour tester l'intégrité des lignes d'aluminium des circuits intégrés lorsque ceux-ci ont été soumis à des cycles thermiques. Là encore, compte tenu de la très faible épaisseur des lignes (1 à $2 \mu \mathrm{m})$, une très faible perturbation (équivalente à quelques nm d’air) sera facilement observable.

Soulignons enfin que ces méthodes photothermiques ne sont pas limitées à l'étude de défauts ou de structures parallèles à la surface mais que des délaminations perpendiculaires (ou inclinées) vont également modifier la distribution de température locale que l'on peut induire avec une source quasi ponctuelle (faisceau focalisé). 
En utilisant le microscope photothermique, la détection infrarouge ou la détection mirage, différents "défauts" ont pu être quantifiés : résistances thermiques entre grains dans les céramiques, microfissures dans les structures métalliques, contrôle de la qualité du front de soudure, etc.

À titre d'exemple l'amplitude et la phase du signal photothermique au voisinage d'une fissure sont représentées sur la figure 8 . La détection se fait à l'aide du montage de la figure 4 qui utilise un détecteur infrarouge double à $10 \mu \mathrm{m}$.

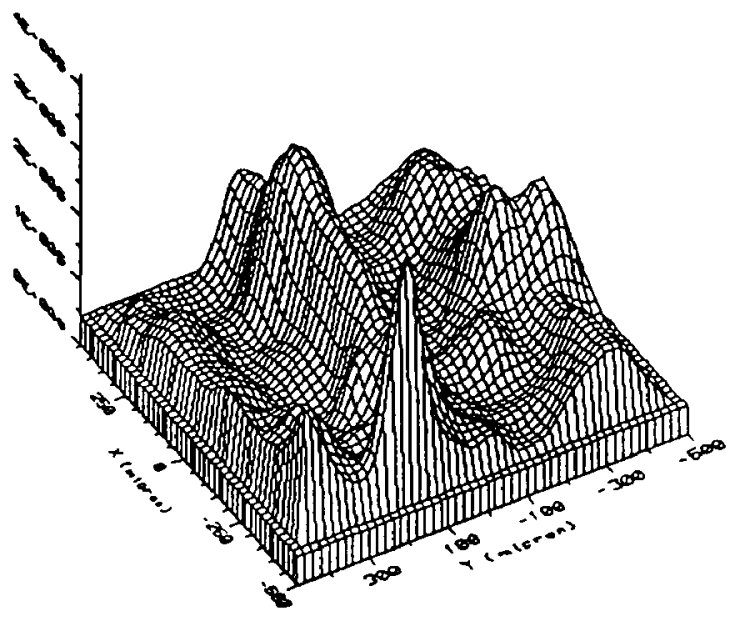

Figure 8a : Amplitude du signal en bidétection lors du balayage en surface d'un échantillon d'acier présentant une fissure inclinée de $20^{\circ}$ par rapport à la normale à la surface, parallèle à l'axe des $\mathrm{X}$.

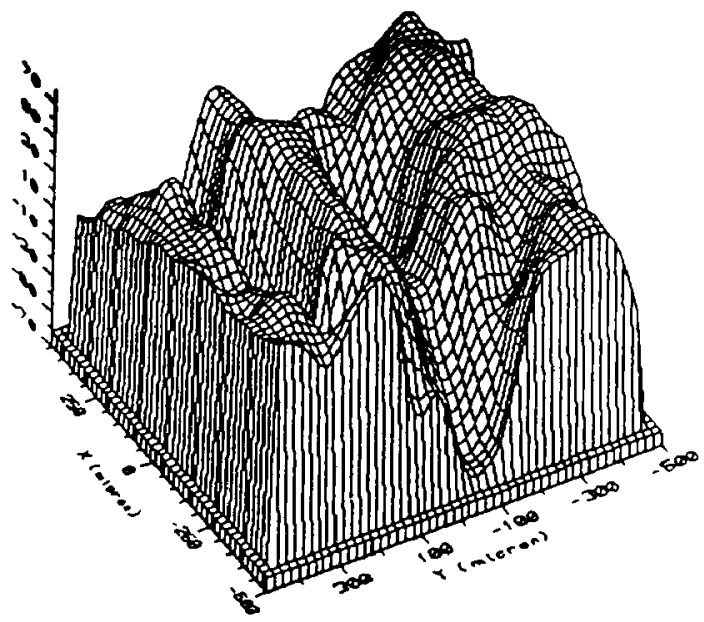

Figure 8b : Phase du signal en bidétection lors du balayage en surface d'un échantillon d'acier présentant une fissure inclinée de $20^{\circ}$ par rapport à la normale à la surface, parallèle à l'axe des $X$. 


\section{LES MESURES D'ABSORPTION ET LA SPECTROSCOPIE PHOTO- THERMIQUE}

La détection photothermique révèle la part de l'énergie lumineuse absorbée et convertie en énergie thermique. Nous savons qu'à quelques exceptions près (matériaux fluorescents, photodiodes...) ce processus de dégradation de l'énergie est prépondérant et qu'il faut chercher les rares situations pour lesquelles les processus optiques, photochimiques ou photovoltaïques sont dominants.

Peut-on utiliser les signaux des détections photothermiques pour mesurer des coefficients d'absorption et dans quelle mesure ces méthodes doivent-elles se substituer aux mesures photo-électriques classiques?

La réponse est qu'il ne faut utiliser les méthodes photothermiques que lorsque les méthodes traditionnelles qui mesurent le flux incident $\phi_{0}$ et le flux émergent $\phi$ sont inutilisables. En particulier :

- lorsque l'échantillon est opaque $(\phi=0)$, il n'est pas possible de mesurer le flux émergent,

- lorsque l'échantillon est trop peu absorbant, la différence $\phi_{0}-\phi$ est difficilement mesurable (typiquement lorsque $\phi_{0}-\phi \ll 10^{-3} \phi_{0}$ ).

Dans les autres cas les méthodes photo-électriques demeurent les plus simples et les plus performantes.

\section{1 Échantillons opaques}

La figure 9 représente un échantillon que nous supposerons homogène, de coefficient d'absorption $\alpha$ et dont la longueur de diffusion thermique à la fréquence f de l'expérience a pour valeur $\mu$.

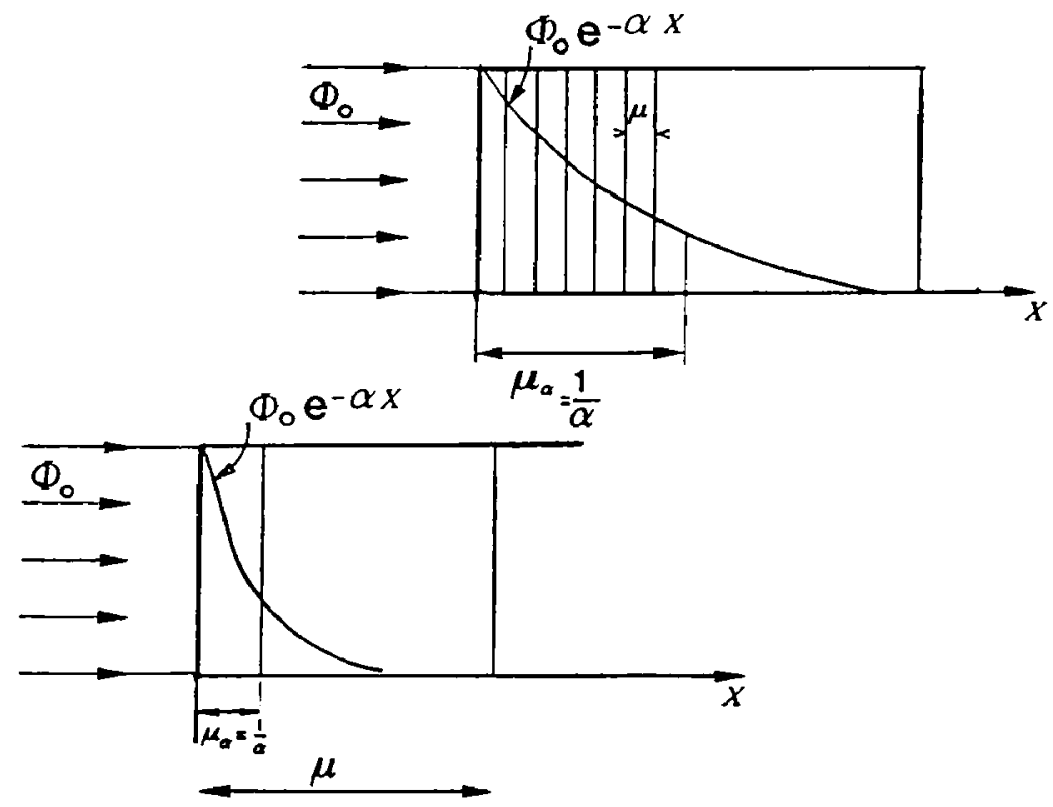

Figure 9 : Spectroscopie photothermique d'échantillons opaques. 
Pour calculer la température de surface, il nous faut écrire les équations de diffusion de la chaleur dans l'air et dans l'échantillon et les conditions aux limites aux interfaces. Ce calcul simple mais un peu long est très bien décrit dans le livre de Rosencwaig; aussi, nous contenterons-nous de décrire semi-quantitativement les effets, dans deux cas limites importants. Rappelons que la longueur de diffusion thermique $\mu$ représente sensiblement la longueur sur laquelle la chaleur diffuse pendant une période de modulation, aussi seul le bilan de l'énergie au sein de la couche d'épaisseur $\mu$ la plus voisine de la surface est-il nécessaire.

L'énergie par unité de surface envoyée sur l'échantillon pendant une période est environ $\phi_{\mathrm{O}} / 2 \mathrm{f}$. L'énergie absorbée dans la première couche d'épaisseur $\mu$ aura pour valeur $\left(\phi_{\mathrm{O}} / 2 \mathrm{f}\right)$ $(1-\exp (-\alpha \mu))$.

Si $\mu \ll<\frac{1}{\alpha}$ on aura un échauffement $\Delta \mathrm{T} \approx \frac{\phi_{0}}{2 \mathrm{f}} \frac{\alpha}{\rho \mathrm{c}}$ et le signal thermique sera bien proportionnel au coefficient d'absorption $\alpha$. On a en quelque sorte découpé l'échantillon en tranches d'épaisseur $\mu$.

Si $\mu>\frac{1}{\alpha}$ on voit que, quel que soit $\alpha$, toute l'énergie sert à chauffer la première tranche d'épaisseur $\mu$; l'élévation de température $\Delta T \approx \frac{\phi_{0}}{2 \mathrm{f}} \frac{1}{\mu \rho c}$ est alors indépendante de $\alpha$, on dit que le signal photothermique est saturé.

Le passage d'un régime à l'autre s'effectuant de façon progressive, il faut veiller à ne pas saturer les parties les plus absorbantes du spectre. Le contrôle peut se faire en augmentant la fréquence de modulation ce qui réduit la longueur de diffusion thermique $\mu$, ou en suivant la phase du signal (on peut montrer qu'il existe un écart de phase de $45^{\circ}$ entre un signal saturé et un signal qui ne l'est pas).

A titre d'exemple la figure 10 représente le spectre d'une poudre d'oxyde d'holmium obtenu avec un monochromateur.

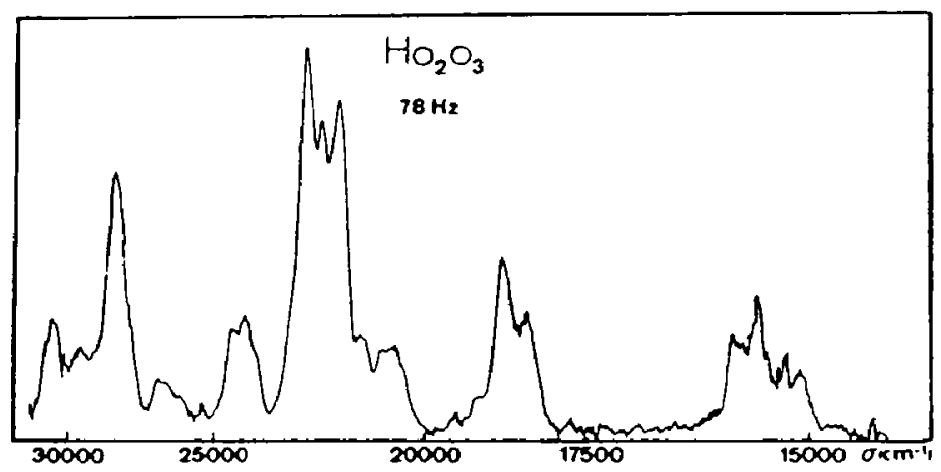

Figure 10 : Spectre photothermique d'une poudre d'oxyde d'holmium.

La détection photothermique étant limitée en sensibilité par le bruit propre du "détecteur thermique" constitué par l'échantillon et le montage à effet mirage par exemple, on a intérêt à utiliser toutes les méthodes de spectroscopie qui se sont révélées si utiles dans ce cas, comme la spectroscopie par Transformation de Fourier pour profiter pleinement du multiplexage spectral et du gain en flux. 
Nous avons réalisé un interféromètre UV - visible - proche IR (3000 à $\left.30000 \mathrm{~cm}^{-1}\right)$ capable de fournir près d'un watt de puissance lumineuse pour une résolution de $16 \mathrm{~cm}^{-1}$ avec lequel de très nombreux spectres de terres rares ou de semiconducteurs amorphes ont été obtenus.

La figure 11 montre un détail du spectre photothermique de l'oxyde d'holmium et la comparaison avec le spectre obtenu avec un laser à colorant.

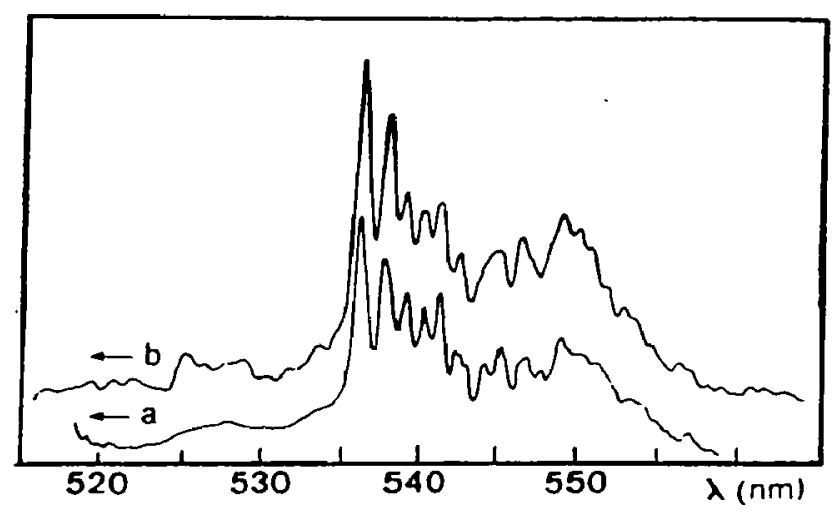

Figure 11 : Comparaison du spectre photothermique de l'oxyde d'holmium obtenu avec un laser à colorant (a) et avec l'interféromètre à transformation de Fourier (b).

Comme dernier exemple de spectroscopie photothermique nous voudrions montrer que cette méthode peut aussi effectuer une discrimination en profondeur des centres absorbants (un peu comme l'ellipsométrie mais à une échelle très différente et éventuellement sur des échantillons optiquement "sales", à la différence des couches minces pour l'optique et la micro-électronique).

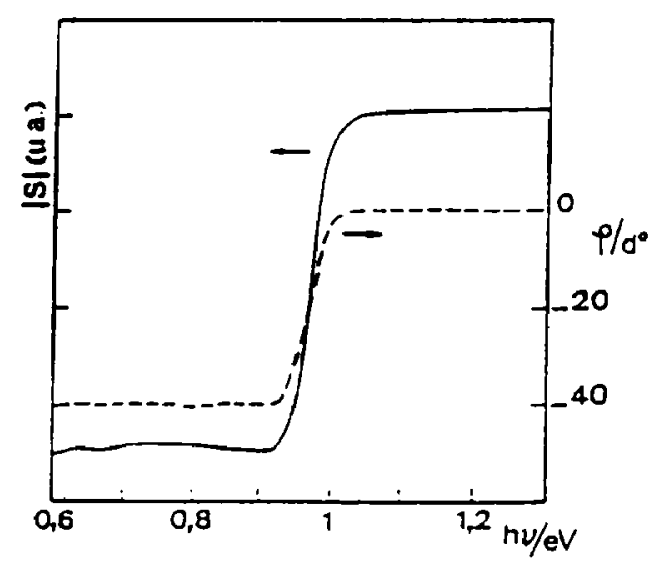

Figure 12 : Spectre du module et de la phase du signal photothermique pour le monocristal de CulnSe 2. 
Considérons la figure 12. Elle représente les spectres photothermiques en amplitude et en phase d'un cristal de $\mathrm{CuInSe} 2$ au voisinage de la bande interdite .

- À haute énergie, le matériau absorbe fortement (d'où la forte amplitude) et la chaleur peut diffuser vers la surface et être détectée avec une phase que nous prendrons comme référence.

- À basse énergie, seul le signal venant de la couche superficielle est capable de chauffer, aussi n'observe-t-on qu'une absorption faible et déphasée de $45^{\circ}$ ce qui permet de confirmer que cette absorption vient bien de la surface.

Cette aptitude des méthodes photothermiques, unique à notre connaissance, permet donc de réaliser une analyse en profondeur d'échantillons hétérogènes ; elle s'appuie bien sûr sur une modélisation, parfois complexe, lorsque le matériau contient plusieurs couches dont les propriétés optiques et thermiques sont différentes.

\subsection{Echantillons très faiblement absorbants}

L'intérêt des méthodes photothermiques pour ce type d'échantillons tient au fait qu'il est difficile de mesurer avec un détecteur photo-électrique la très faible différence entre le flux incident et la somme des flux transmis, diffusés, réfléchis pour connaître le flux absorbé. Il paraît en effet plus logique de mesurer directement l'élévation de température résultant de l'énergie absorbée par l'échantillon.

A quelles classes de problèmes s'adresse ce type de mesures?

Pour ce qui est des matériaux en phase condensée, la fabrication d'échantillons diélectriques présentant de très faibles absorptions est un enjeu important :

- On sait par exemple que les pertes par absorption des meilleures fibres optiques utilisées en télécommunication dans le proche infrarouge sont inférieures au $\mathrm{dB} / \mathrm{km}$.

- Les traitements diélectriques des miroirs ou des antireflets utilisés pour les lasers de puissance ou pour des interféromètres de très grande finesse présentent une absorption qui se situe à un niveau inférieur à une partie par million de l'énergie incidente.

Pour mesurer de tels niveaux d'absorption dans la silice ou dans d'autres matériaux pour l'optique non linéaire, nous utilisons les méthodes photothermiques.

Les très faibles absorptions dans les gaz permettent de nombreuses applications comme l'analyse de composés à très faible concentration, ou de la pollution atmosphérique. Ces thèmes porteurs ont déjà fait un usage important des détections photothermiques.

\subsection{1 Échantillons en couches minces.}

Dans ce cas le substrat est généralement transparent et l'énergie absorbée dans la couche superficielle (ou l'empilement) peut être mesurée par la méthode mirage décrite précédemment.

L'échantillon est le plus souvent immergé dans un liquide $\left(\mathrm{CCl}_{4}\right.$, huile transparente...) pour augmenter le facteur dn/dT et donc le signal mesuré.

Cette méthode a été employée par de nombreux laboratoires pour explorer les états du "pseudo-gap" des semiconducteurs amorphes et en particulier du silicium amorphe hydrogéné.

Pour certains échantillons, nous avons pu mesurer des densités optiques variant sur 6 ordres de grandeur, aussi faut-il souligner la très grande dynamique que peuvent présenter de telles mesures.

Lorsque l'on dispose de sources cohérentes pour irradier l'échantillon, il est souvent plus efficace de profiter du gradient qui peut être créé en focalisant le laser de chauffe à la su.face de l'échantillon. Dans ce cas, selon la fréquence de modulation, la distribution thermique sera fixée par la taille et la structure du faisceau laser (à haute fréquence la distribution de température suivra celle de l'intensité du mode gaussien par exemple) ou par la diffusion thermique (à basse fréquence la distribution sera dominée par la diffusion si la longueur de diffusion thermique est plus grande que le diamètre du faisceau). La "lentille thermique" ainsi 
créée dans l'air, dans la couche et dans le substrat, peut être sondée par un faisceau sonde placé dans la zone de gradient maximum. Grâce à la grande densité d'énergie dont on dispose sur l'échantillon, on obtient une très bonne sensibilité (Figure 13).

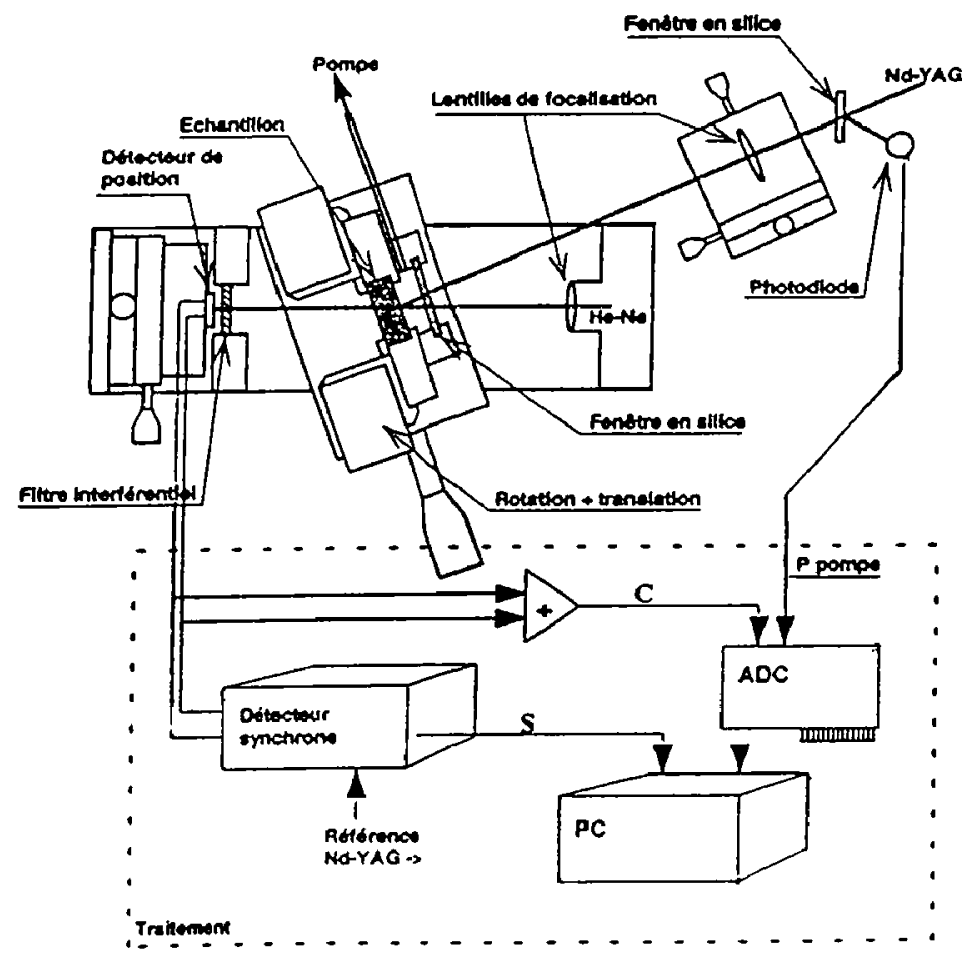

Figure 13 : Schéma du banc mirage pour les traitements de surface : sensibilité $7 \cdot 10^{-9}$ en absorption.

Par exemple, le montage que nous utilisons pour mesurer les pertes par absorption des miroirs du projet VIRGO (Antenne Interférométrique pour la Détection d'Ondes Gravitationnelles) nous permet d'atteindre une sensibilité équivalente à l'absorption de quelques parties par milliard de la lumière incidente (avec 1 watt de lumière laser à $1,06 \mu \mathrm{m}$ et des substrats de silice).

\subsection{2 Échantillons massifs.}

Dans ce cas la géométrie colinéaire s'impose car on a intérêt à avoir le maximum de recouvrement entre le faisceau de chauffe et le faisceau sonde. Le montage de la figure 14 est celui qui sert à tester les matériaux des optiques de VIRGO qui seront traversées par la lumière (lame séparatrice, miroirs d'entrée, lame de recyclage). Remarquons qu'il est possible de disposer de miroirs de très faible absorption pour replier le faisceau YAG sans craindre des signaux parasites dus à l'échauffement (donc à la déformation) de ces composants.

La colinéarité des faisceaux n'est pas totale car on cherche à éviter les signaux qui pourraient provenir d'une éventuelle pollution des surfaces ; aussi la pompe et la sonde sontelles décalées sur les faces d'entrée et de sortie de l'échantillon. 
Nous avons trouvé une très grande diversité des niveaux d'absorption dans la silice et quelques cristaux réputés "transparents".

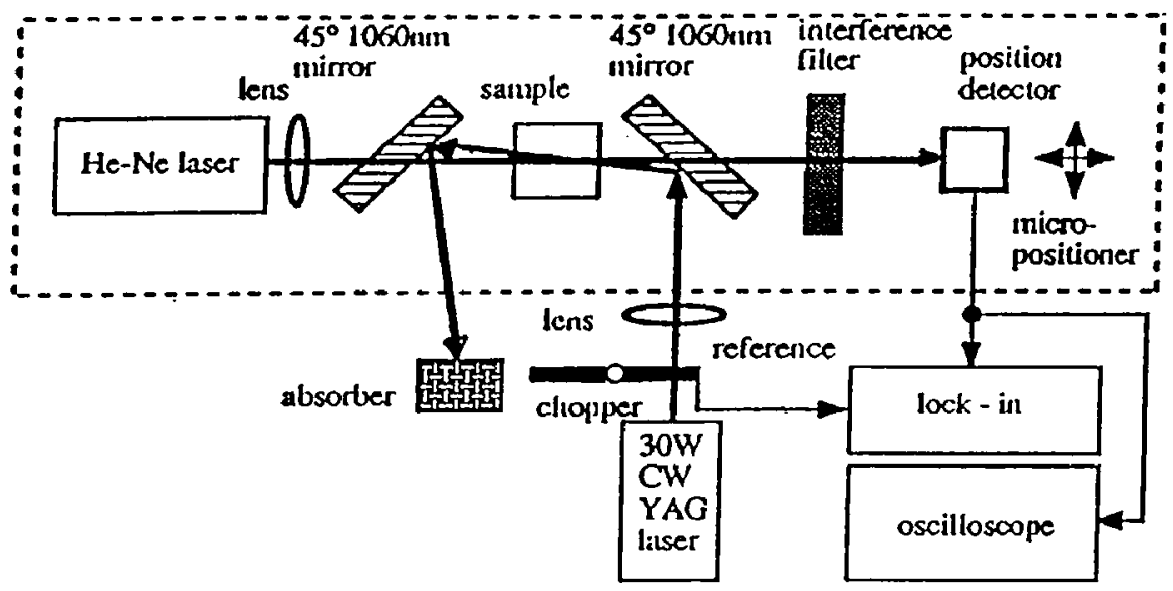

Figure 14 : Mesure de pertes par absorption par effet mirage pour un matériau massif.

Une étude récente sur des échantillons de "saphir" ( $\mathrm{Al}_{3} \mathrm{O}_{3}$ monocristallin) que l'on sait aujourd'hui préparer en grandes dimensions avec une excellente pureté (principalement en ions du groupe du fer comme le $\mathrm{Cr}^{3+}$ ) a montré les progrès réalisés dans le domaine puisque les pertes sont actuellement de l'ordre de $10^{-6} \mathrm{~cm}^{-1}$.

Ce type de montage nous a également permis de mesurer l'absorption dans des matériaux pour l'optique non linéaire. On sait que ces matériaux sont soumis à de très grandes densités d'énergie (et/ou de puissance) et leur absorption résiduelle peut induire la déformation des fronts d'ondes ou être à l'origine de la destruction du matériau. La seule précaution qu'il faut prendre sur ces milieux anisotropes est de bien contrôler la polarisation des faisceaux sonde et pompe, car tous les paramètres physiques en dépendent (absorption, dn/dT...) lorsqu'on fait une expérience.

\subsubsection{Gaz.}

La détection mirage "colinéaire" se prête bien à la mesure de très faibles absorptions en phase gazeuse. De nombreuses applications au contrôle de l'environnement (dosage de polluants atmosphériques) et à l'analyse chimique peuvent être menées à bien avec cette technique.

Le montage utilisé (figure 15) permet de placer le faisceau de chauffe (Laser à $\mathrm{CO}_{2}$ accordable) et le faisceau sonde (Laser $\mathrm{He} / \mathrm{Ne}$ ) parallèlement l'un à l'autre sur une distance d'une dizaine de $\mathrm{cm}$ (distance entre les fenêtres de Ge à l'incidence de Brewster du laser à $\mathrm{CO}_{2}$ ).

Le laser à $\mathrm{CO}_{2}$ fonctionne sur une centaine de raies dans la zone 9 à $11 \mu \mathrm{m}$. La séparation entre chaque raie est de l'ordre $\mathrm{du} \mathrm{cm}^{-1}$ (niveau de rotation) et la coïncidence entre ces raies et celle des molécules qui absorbent dans cette région du spectre fournit une empreinte typique du gaz à détecter. 


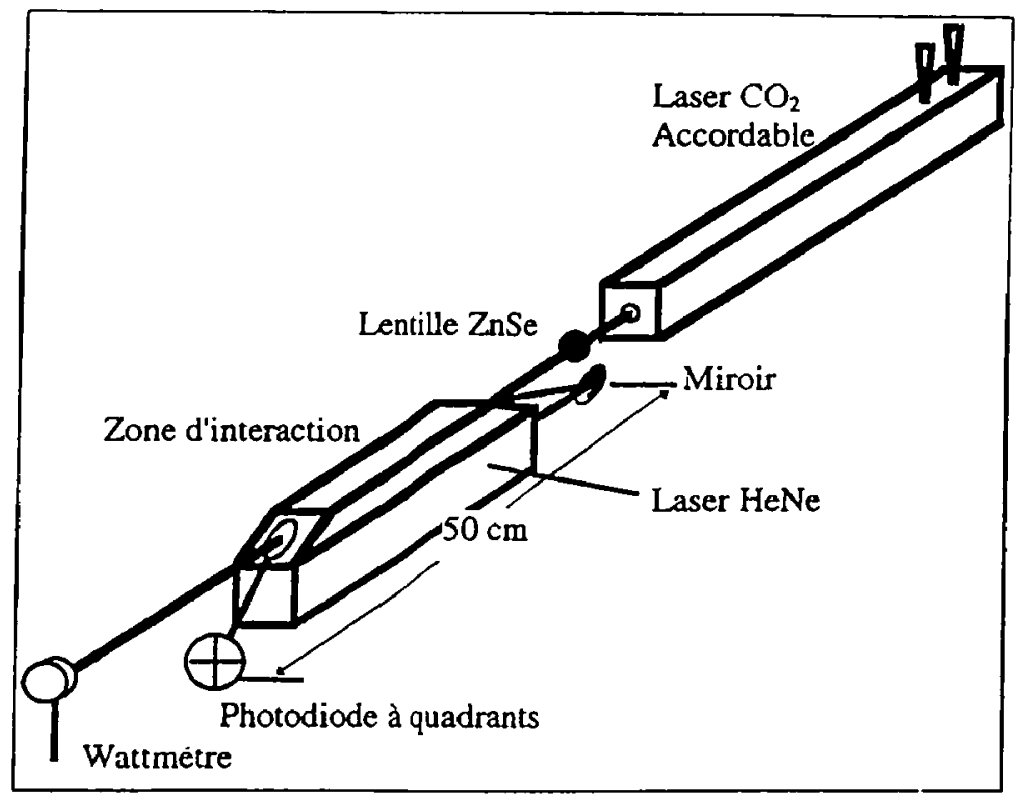

Figure 15 : Montage par effet mirage colinéaire pour la détection de gaz.

- A titre d'exemple la figure 16 montre les signaux obtenus pour l'éthylène $\mathrm{C}_{2} \mathrm{H}_{4}$ autour de la raie $\mathrm{P}_{14}$ à $10,6 \mu \mathrm{m}$. Cette étude montre que le niveau minimum de $\mathrm{C}_{2} \mathrm{H}_{4}$ détectable dans l'air à la pression atmosphérique est de 0,1 partie par milliard ce qui correspond à une absorption minimum détectable de $310^{-9} \mathrm{~cm}^{-1}$ (remarquons que le gain en sensibilité de deux ordres de grandeur par rapport aux solides est principalement dá au rapport $(\mathrm{dn} / \mathrm{dT}) / \mathrm{p}$ ).

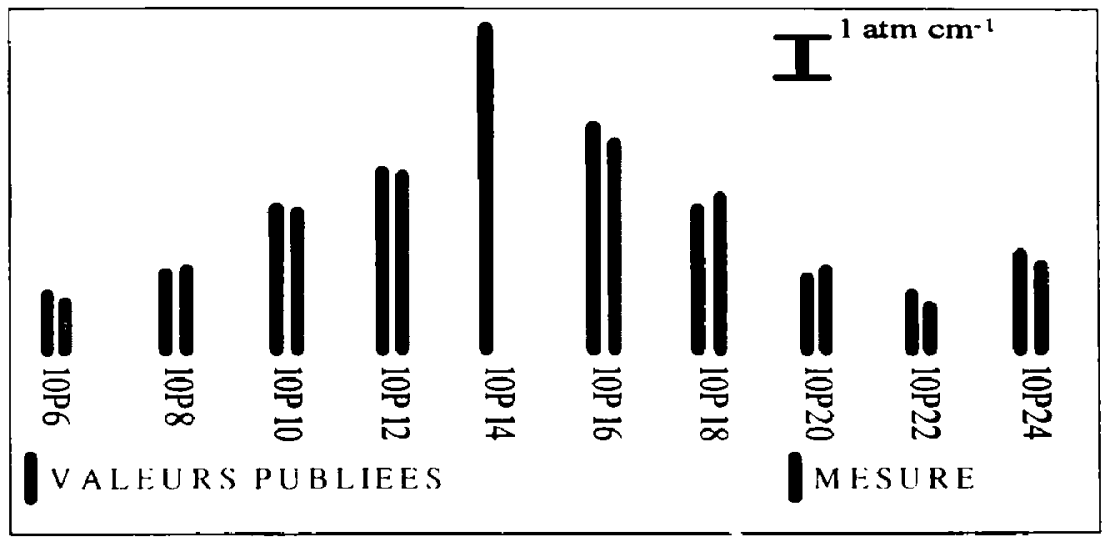

Figure 16 : Signaux obtenus par effet mirage sur un mélange de 50 ppm d'ethylène dans l'azote. 


\section{CONCLUSION}

A travers les quelques exemples d'instruments de métrologie ou de caractérisation que nous venons de décrire, nous avons cherché à illustrer quelques uns des avantages des sondes optiques :

- sondes non destructives sans contact matériel avec l'échantillon.

- sondes sensibles souvent limitées dans leur performances par des bruits fondamentaux.

Nous avons pris le prétexie des méthodes photothermiques pour introduire des phénomènes qui peuvent fournir à la fois des paramètres thermiques (diffusivité) et des paramètres optiques (absorption) sur des échantillons le plus souvent exotiques.

Il va de soi que les schémas expérimentaux, très classiques, présentés ici pourront utilement être transposés à de nombreuses classes de problèmes et qu'ils pourront contribuer à la diffusion des méthodes optiques dans les domaines les plus variés.

\section{Bibliographie}

Pour faciliter l'approche d'un sujet pour lequel les très nombreuses publications se dispersent sur beaucoup de journaux nous conseillons la lecture des ouvrages de synthèses et les comptes rendus des congrès internationaux.

\section{Ouvrages de synthèse}

- Pao Y.H. Ed., Optoacoustic Spectroscopy and Detection (Academic Press 1977)

- Rosencwaig A., Photoacoustics and Photoacoustic Spectroscopy (John Wiley and Sons 1980)

- Mandelis A. Ed., Photoacoutic and thermal waves in semiconductors (North Holland 1987)

- Hess P. Ed., Photoacoustic, Photothermal and Photochemical Processes at Surfaces and Thin Films (Topics in current Physics Springer Verlay 1989)

- Sell J.A. Ed.,Photothermal Investigation of Solids and Fluids (Academic Press 1989)

- Bialkowski S.E., Photothermal Spectroscopy Methods for chemical Analysis (John Wiley and Sons 1996)

\section{Comptes rendus de congrès}

- Fournier D. et Badoz J. Eds., Third International Meeting on Photoacoustic and Photothermal Spectroscopy (1983), Journal de Physique, C6

- Bertrand L. Ed., Proceedings of the 4th Topical Meeting on Photoacoustics Thermal and Related Sciences, Journal Canadien de Physique, 64 (1986) $n^{\circ} 9$

- Hess P. and Pelzl J. Eds, Photoacoustic and Photothermal Phenomena, Springer Series In Optical Sciences, 1988

- Murphy J.C. et al Eds, Photoacoustic and Photothermal Phenomena II, Springer Series In Optical Sciences 1990

- Bicanic D. Ed.. Photoacoustic and Photothermal Phenomena III, Springer Series In Optical Sciences 1992

- Fournier D. and Roger J.P., 8th International Topical Meeting on Photoacoustic and Photothermal Phenomena, Joumal de Physique C7 1994 